\title{
Automatic Calibration Using a Modified Genetic Algorithm for Millimeter-Wave Antenna Modules in MIMO Systems
}

\author{
Cheng-Nan Hu $\mathbb{D}^{\mathrm{D}},{ }^{1}$ Philip Lo, ${ }^{2}$ Chien-Peng Ho, ${ }^{1}$ and Dau-Chyrh Chang ${ }^{1}$ \\ ${ }^{1}$ Communication Engineering Department of OIT, New Taipei City 22061, Taiwan \\ ${ }^{2}$ FIH Limited, FTC Division, No. 4, Minsheng Street, Tucheng District, New Taipei City, Taiwan \\ Correspondence should be addressed to Cheng-Nan Hu; fo011@mail.oit.edu.tw
}

Received 25 November 2019; Revised 24 February 2020; Accepted 10 April 2020; Published 3 June 2020

Academic Editor: Giuseppe Castaldi

Copyright (c) 2020 Cheng-Nan Hu et al. This is an open access article distributed under the Creative Commons Attribution License, which permits unrestricted use, distribution, and reproduction in any medium, provided the original work is properly cited.

\begin{abstract}
This study proposes a method for designing and calibrating a millimeter-wave ( $\mathrm{mm}$-wave) multiple-input multiple-output (MIMO) antenna module. Herein, we adopt a design example involving a 64-element MIMO antenna array arranged in a triangular lattice (instead of the commonly used rectangular lattice) to achieve a $3^{\circ} \mathrm{dB}$ enhancement in effective isotropic radiated power. Analyzing a grating lobe diagram indicates a scan volume of $\pm 60^{\circ} / \pm 45^{\circ}$ in the azimuth/elevation direction. To calibrate the massive mm-wave MIMO antenna module, we propose a modified genetic algorithm to align the amplitude/phase of the transmitting/receiving signal of the module to reduce the time required for the calibration process. Finally, we conducted a simple experiment to validate the proposed method.
\end{abstract}

\section{Introduction}

Fifth-generation mobile communication technology is expected to introduce an advanced air interface in new radiofrequency bands. Millimeter-wave (mm-wave) frequency bands (i.e., $\geq 26 \mathrm{GHz}$ ) can provide a channel bandwidth of approximately $1 \mathrm{GHz}$ (or higher) to enhance mobile broadband function with a throughput of up to $10 \mathrm{~Gb}$, can support ultrareliable and low-latency communication capability with submillisecond latency under required packet loss, and can enable massive machine-type communication to connect billions of devices (i.e., Internet of Things (IoT)) [1]. Large-scale antenna arrays (also called multiple-input multiple-output (MIMO) arrays) with beam-forming technology for single-user MIMO or multiple-user MIMO communication between a base station and mobile devices can provide high antenna gain to overcome radiation propagation path losses at the aforementioned frequencies. Moreover, sustaining an effective radio link budget can engender high-data-rate communications and high-capacity operation in networks. The effective isotropic radiated power (EIRP) is a crucial measure of transmitter performance.
Herein, we adopt a design example involving a 64-element MIMO array arranged in a triangular lattice to increase the antenna gain by $3 \mathrm{~dB}$.

To equip a massive large MIMO system with beamsteering capability, the system must be adequately calibrated to generate the desired phase front to steer a dynamic threedimensional pencil beam in a specified direction. Consider, for example, MIMO antennas. After the radiating signals of the MIMO antennas are adequately calibrated and aligned uniformly across the antenna aperture, the antennas can generate a pencil beam pattern with a very high EIRP in the boresight direction; thus, the signal quality can be improved for high-data-rate applications.

Massive MIMO arrays can be calibrated through either over-the-air (OTA) field tests, including those that involve far-field [2-5] and near-field [6-8] test ranges, or built-in self-calibration tests [9]. Near-field techniques are advantageous for calibrating large-scale phased array systems [6] because the techniques provide useful information to antenna designers; specifically, the techniques provide information that is useful for diagnosing antenna illumination through the back-transformation of the computed far-field 
value to the aperture of the AUT using an inverse fast Fourier transform. However, the signal level detected in the nearfield range is susceptible to noise interference, especially in $\mathrm{mm}$-wave systems. In built-in self-calibration tests, phase toggling is applied to implement a closed-loop transmitting/ receiving coupler near the radiating element for signal detection. Although detected signals can be used for monitoring, calibration, and fault isolation in phased antenna array systems [9], signal deviations caused by parasitic circuit effects of the radiated structure, feeding network, and radome (i.e., housing of the system) cannot be considered in $\mathrm{mm}$-wave systems. Moreover, the array systems must have a sufficient built-in circuit design for closed-loop testing and calibration to ensure adequate operation. This can be achieved using radio-frequency integrated circuit (RFIC) technology for a medium-sized array system with low-power operation. However, such an arrangement is complex for an array with large antenna elements and high-power operation.

To solve the aforementioned MIMO calibration problems, this study proposes a calibration method that entails first applying a modified genetic algorithm (GA) in the farfield test range to calibrate an mm-wave massive MIMO system. The advantages of the proposed calibration method are as follows:

(1) No additional closed-loop built-in circuits are required, thus reducing system design complexity

(2) The overall system calibration is considered to ensure accuracy

(3) The modified GA with machine learning capability based on big data can be easily applied to a mass production line for automatically calibrating 5G massive MIMO systems

The remainder of this paper is organized as follows. Section 2 presents the theoretical approach of the proposed method and the numerical investigation of the simulated results. In Section 3, a simple experimental study is reported. Section 4 provides the study conclusion.

\section{Theory and Numerical Results}

2.1. System Configuration. Figure 1 illustrates the design concept of an mm-wave massive MIMO antenna module that can automatically undergo self-calibration through the modified GA. The MIMO antenna module comprises the following components: (1) 64-element microstrip patch antenna array; (2) 16 front-end modules, each of which has four-channel transmit/receive (TR) devices; (3) a power distribution network; and (4) a test antenna in the far-field range. This study adopted a four-element TRX half-duplex silicon front-end IC (F5280, IDT Inc.) [10], operating at 25-31 GHz. As presented in Figure 1(b), the antenna array comprises a set of polarized elements with position vector $D=\left[d_{1}, d_{2}, \ldots, d_{m}, \ldots, d_{M}\right]$, where $d_{m}$ is the location of the $m$ th antenna element.

The far-field pattern of the simultaneously excited MIMO antenna array is expressed as follows:

$$
f(\theta, \varphi)=W^{H} \cdot e^{-j K^{T} \cdot D} \xi(\theta, \varphi)=W^{H} \cdot E(\theta, \varphi),
$$

where

$$
\begin{aligned}
W & =\left[w_{1}, w_{2}, \ldots, w_{M}\right]^{T}, \\
E(\theta, \varphi) & =e^{-j K^{T} \cdot D} \xi(\theta, \varphi), \\
K & =\frac{2 \pi}{\lambda}[\sin \theta \cos \varphi \sin \theta \sin \varphi \cos \theta]^{T},
\end{aligned}
$$

where $\lambda$ is the wavelength at frequency $f ; \theta$ and $\phi$ are the azimuthal angle and angle of elevation of the far-field source, respectively; and $T$ is the matrix transpose.

$\xi(\theta, \varphi)$ is a function of the polarized pattern of an individual element, and $w_{i}\left(w_{i}=c_{i} e^{j \psi_{i}}\right)$ is the complex weighting coefficient at the $i$ th element of weight vector $\mathbf{W}$. The Hermitian transpose, which combines transposition and conjugation, is denoted by $\mathbf{H}$, and the radiation pattern of the array is denoted by $f(\theta, \varphi)$. To satisfy the scan volume requirement, the spacing arrangement of the antenna elements in the system should be specified, that is, either a rectangular or triangular lattice. For a rectangular lattice, the spacings in the $x$-axis, $y$-axis, and $\mathrm{d} x / \mathrm{d} y$ can be expressed as follows:

$$
\begin{aligned}
& \mathrm{d} x \leq \frac{\lambda}{1+\sin \left(\theta_{a z}\right)}, \\
& \mathrm{d} y \leq \frac{\lambda}{1+\sin \left(\theta_{e l}\right)},
\end{aligned}
$$

where $\theta a z / \theta e l$ is the scan volume specification in the azimuth/elevation direction.

Through (4) and (5), the $\mathrm{d} x$ and $\mathrm{d} y$ values for elements arranged in a rectangular lattice can be derived as $0.536 \lambda$ and $0.586 \lambda$, respectively, satisfying the scan coverage requirement of $\mathrm{az}=60^{\circ}$ and $\mathrm{el}=45^{\circ}$. Moreover, for elements arranged in a triangular lattice, the $\mathrm{d} x$ and $\mathrm{d} y$ values can be derived as $1.0733 \lambda$ and $0.6066 \lambda$, respectively, by analyzing a grating lobe diagram [11], as shown in Figure 1(c), where the solid and dashed lines represent the boundary of the scan volume and grating lobe, respectively. Since the aperture size is increased by arranging array elements in a triangular lattice, the MIMO provides relatively high antenna gain under the same scan volume requirement; nevertheless, such an increase is not suitable for miniaturization. On the basis of the derived spacing values, the maximum element gain $\left(G e=4 \pi \mathrm{d} x \mathrm{~d} y / \lambda^{2}\right)$ corresponding to the rectangular and triangular lattice arrangements can theoretically be computed to be 5.96 and $9.13 \mathrm{dBi}$, respectively. This thus indicates that the EIRP is theoretically improved by approximately $3 \mathrm{~dB}$. This improvement is crucial to MIMO design because power amplification results in poor efficiency in $\mathrm{mm}$-wave systems. The use of additional power amplifiers for a $3 \mathrm{~dB}$ output power gain will increase power consumption levels and engender heat sink problems in such systems. According to the preceding theoretical calculations, the design of massive MIMO systems requires high EIRP in small-cell applications, which reduces volume constraints in space. 


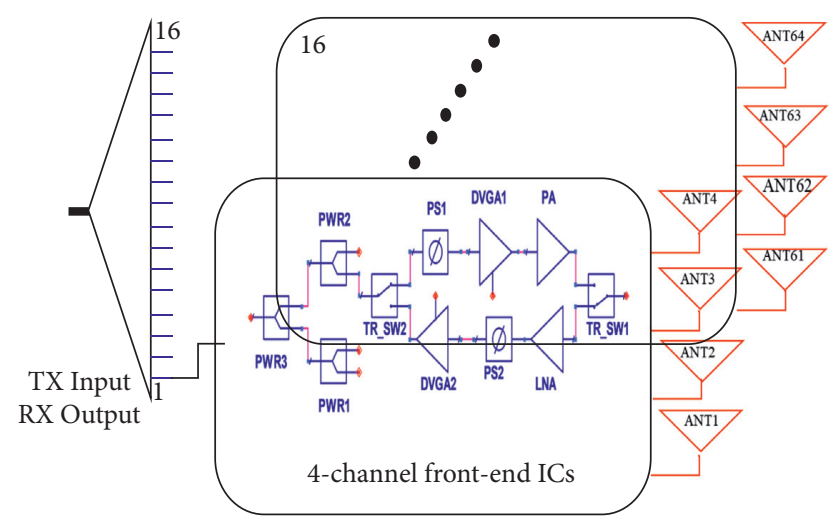

(a)

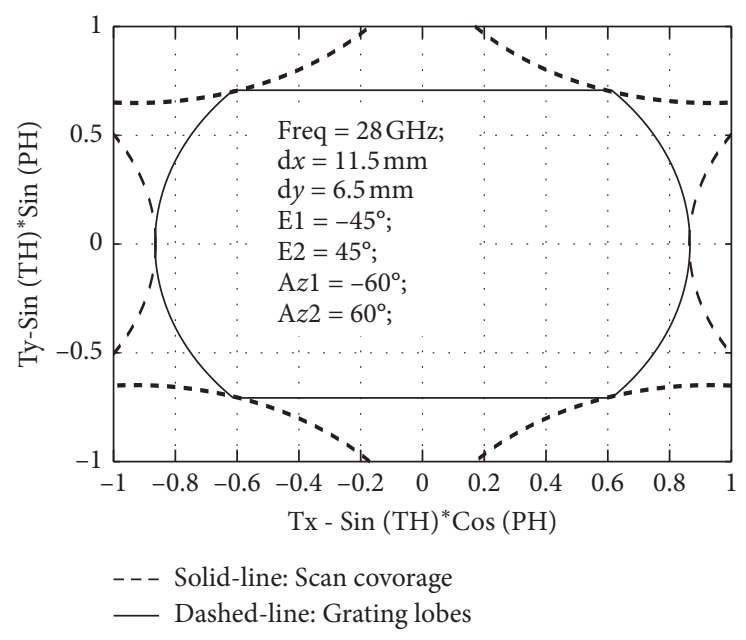

(c)

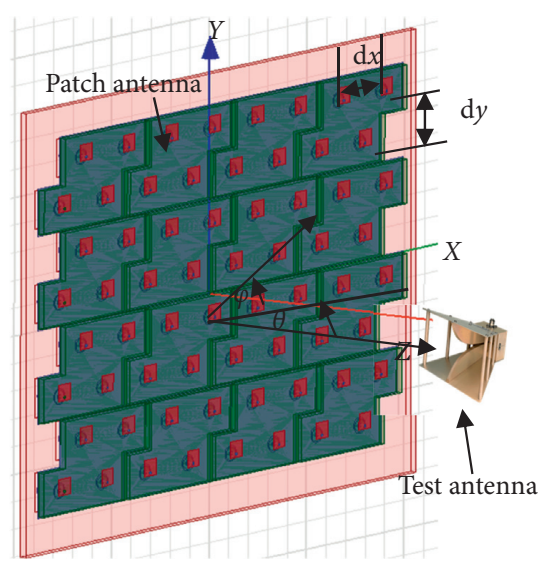

(b)

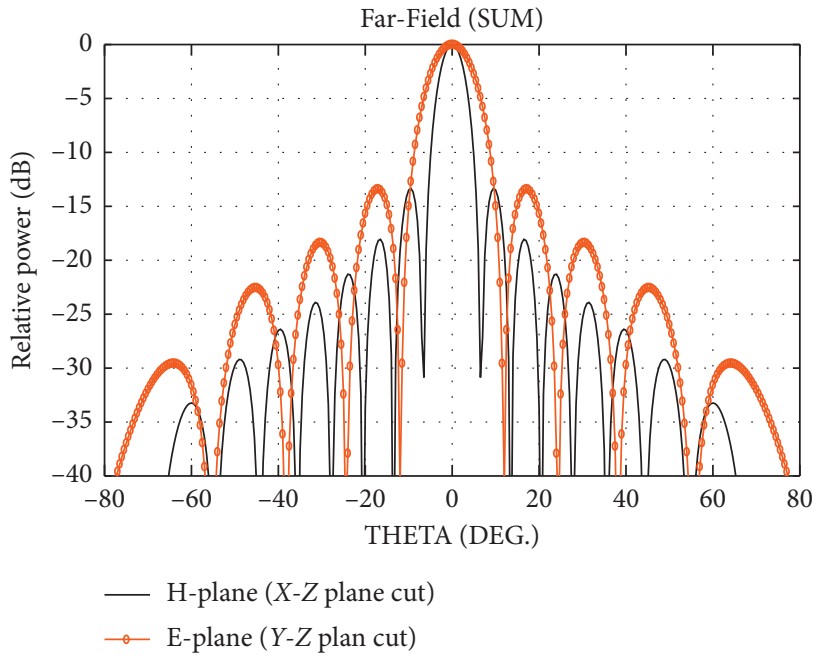

(d)

Figure 1: (a) Schematic of mm-wave active massive MIMO system; (b) $8 \times 8$ element microstrip array $(\mathrm{d} x=11.5 \mathrm{~mm}, \mathrm{~d} y=6.5 \mathrm{~mm})$ associated with a test antenna in the far-field range for calibration; (c) grating lobe diagram analysis (solid line: scan volume, dashed line: grating lobes) of the array with a triangular array lattice of $\mathrm{d} x=11.5 \mathrm{~mm}$ and $\mathrm{d} y=6.5 \mathrm{~mm}$; (d) two-dimensional far-field patterns (E-H plane).

When the excitation coefficient vector $\mathbf{W}$ is calibrated uniformly with equal amplitude and phase distribution, the maximum antenna gain $(\boldsymbol{G} \max )$ can be derived to be $27.19 \mathrm{dBi}$ in the boresight direction $\left(\theta=0^{\circ}\right.$ and $\left.\phi=0^{\circ}\right)$. Calibration is a complex process that involves testing all states of both the phase shifter and the attenuator of each radiating element at various operational frequencies. Consider, for example, a front-end RFIC with 6-bit phase shifters and 4-bit attenuators; calibrating a 64-element MIMO antenna array with 10 frequencies would require approximately $655,360(64 \times 64 \times 16 \times 10=655,360)$ iterations of manual testing per each antenna element.

Accordingly, because of the lack of a built-in closed-loop calibration circuit in front-end RFICs (IDT, F5280) for precise phase and amplitude control of the excitation coefficient vectors, an artificial intelligence calibration method is essential for the automatic calibration of mass-produced massive MIMO systems.
2.2. GA for Far-Field Calibration. On the basis of assumptions or simplifications, engineering problems can be mathematically modeled by imitating real-world principles. Subsequently, mathematical problems can be solved numerically. Finally, the derived problem solution can used to address a broader class of real problems. Several algorithms or systems have been demonstrated to be extremely helpful in addressing problems in various fields, including artificial neural networks [12, 13], fuzzy control [14], simulated annealing $[15,16]$, and GAs. Studies have successfully used GAs for antenna pattern synthesis and autocalibration $[17,18]$ to obtain the optimum excitation coefficient vector $\left(\mathbf{W}_{\text {opt }}\right)$ for achieving high antenna performance (i.e., maximum antenna gain with a specified sidelobe level). Figure 2 presents the general design flow of a GA for the far-field calibration of a massive MIMO in an anechoic chamber; this procedure is used to mathematically imitate the evolution of the excitation coefficient vector (W) iteratively until the 
optimal $\mathbf{W}_{\text {opt }}$ is obtained. The stages in the iterative loop of the GA (Figure 2) are described as follows.

2.2.1. Evaluation. This stage is also called initialization. It entails generating an initial population and its offspring and then ranking genes.

2.2.2. Selection. This stage represents the mechanism for selecting individuals (populations) for reproduction according to their fitness (objective function) values.

2.2.3. Crossover. This stage involves merging the genetic population of two individuals. Two carefully selected parents can produce excellent children.

2.2.4. Mutation. This stage entails making random alterations to gene values. In real evolution, genetic information can be varied randomly by erroneous reproduction or other deformations of genes. In a GA, mutation can be realized as a random deformation of strings with a specified probability.

If the excitation coefficient vector of a MIMO system is adequately calibrated, the maximum measured EIRP would be attained in the boresight direction; this can be approximated as follows:

$$
\begin{aligned}
\text { EIRP } & =G_{A}+10 \log _{10}(N)+P_{\text {elem }}+L+L_{\text {scan }}+\mathrm{SP}, \\
G_{A} & =20 \log _{10}(|f(\theta, \phi)|),
\end{aligned}
$$

where $G_{A}$ is the antenna gain, $P_{\text {elem }}(13 \mathrm{dBm})$ is the transmitter power per element, $N$ is the total number of antenna elements, $\mathrm{SP}(\sim-64.91 \mathrm{~dB})$ is the space loss at a $1.5 \mathrm{~m}$ range and $28-\mathrm{GHz}$ frequency, $L_{\text {scan }}$ is the scan loss $(0 \mathrm{~dB}$ at boresight), and $L(\sim-2 \mathrm{~dB})$ is the total power loss in the antenna. When the weighting vector (W) is adequately calibrated with a uniform distribution, the maximum antenna gain Gmax is close to the antenna's area gain $\left(10 \log _{10}\left(\left(4 \pi A / \lambda^{2}\right)\right) \sim 27.19 \mathrm{~dB} i\right.$; $\mathrm{A}$ is the area of the antenna) in theory. Thus, according to (6), EIRP opt $_{\text {corresponding to a }}$ range $R$ of 0 and $1.5 \mathrm{~m}$ can be estimated to be 56.26 and $-8.65 \mathrm{dBm}$, respectively.

During calibration, the measured EIRP value should be less than the EIRP $_{\text {opt }}$ value because of random amplitude/ phase errors of $\mathbf{W}$ caused by factors such as device variation, unequal transmission line trace lengths, and radome effects. Thus, the cost function $\left(f\left(W_{i}\right)\right)$ at the $i$ th iteration of the GA optimization loop can be defined as follows:

$$
f_{c}\left(W_{i}\right)=\min \left\{\operatorname{EIRP}(i)-\operatorname{EIRP}_{\mathrm{opt}}\right\}
$$

where EIRP ( $i$ ) represents the EIRP measured in the $i$ th iteration of the GA optimization loop for a test antenna in the boresight direction in the far-field range $(R=1.5 \mathrm{~m}$ in this case). When (2) is substituted into (6) and (7), cost function (8) in a root-mean-square sense can be summarized as follows for numerical analysis:

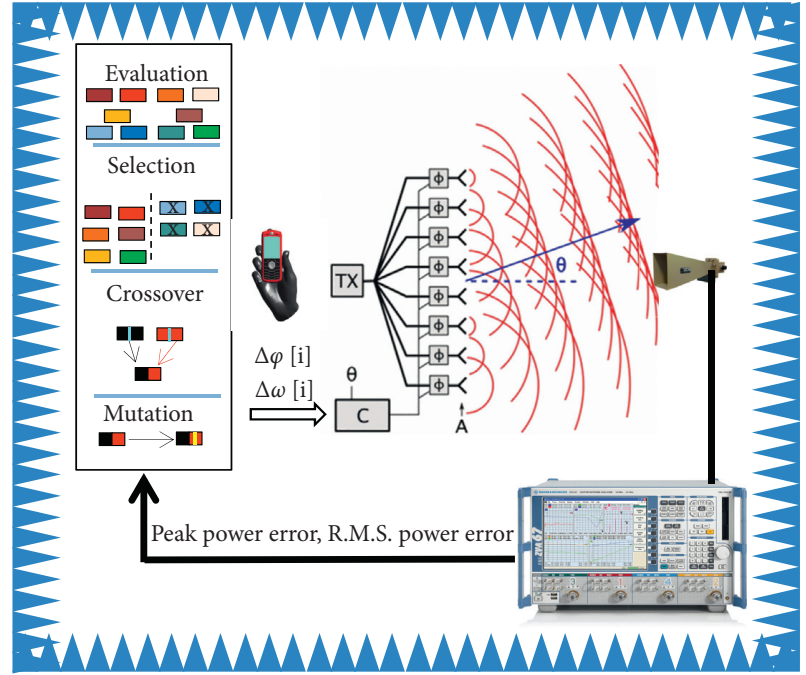

FIGURE 2: Setup of GA for far-field calibration of massive MIMO system in an anechoic chamber.

$$
f_{c}\left(W_{i}\right)=\min \left\{\sqrt{\frac{\left\{\left|\sum_{i=1}^{N} w_{i}\right| / \sum_{i=1}^{N}\left|w_{i}\right|^{2}-N\right\}^{2}}{N}}\right\} .
$$

Accordingly, the GA design flow (Figure 2) can be applied to calibrate the massive MIMO system in order to achieve maximum EIRP and tune the phase and amplitude of $\mathbf{W}$ until the optimal excitation coefficient $\left(W_{\text {opt }}\right)$ is attained. The error of the excitation weighting vector $(\delta W=$ $\left.W_{\text {opt }}-W\right)$ can be computed and written in the internal memory of the front-end ICs to correct the amplitude/phase error of the excitation weighting vectors. Phase errors engendered by the deviation of signal traces connecting from chips to antennas can be considered the determinant errors, but signal errors caused by chip-to-chip variations can be considered random errors, which is the major consideration in the following numerical analysis.

The phase/amplitude of $\mathbf{W}$ is assumed to be randomly distributed uniformly around the interval $(0,2 \pi) /(0.05,0.2)$. The excitation coefficient of each radiating element $\left(\mathbf{W}_{i}\right.$, $i=1,2, \ldots, 64)$ should be calibrated to the normalized value of $0.125(1 / \sqrt{N})$ in order to achieve maximum EIRP or antenna gain. Before the GA iterative loop (Figure 2) is executed for calibration, the excitation coefficient $\left(W_{\mathrm{i}}, i=1\right.$, $2, \ldots, 64$ ) of each element to the binary code should be encoded to represent each population of the generation. In this study, a 6-bit phase shifter associated with three LSBs of the attenuator was used for coding $\mathbf{W}$.

In each population, the phase/amplitude error of the weighting vector $\left(\mathbf{W}_{\boldsymbol{i}}\right)$ is also generated uniformly in the interval $(0,2 \pi) /(0.05,0.2)$ to compute the EIRP (or antenna gain) of each generation using (6) and (8) to rank genes. Subsequently, the inferior genes (poor EIRP) are discarded and superior genes (better EIRP) are crossed over to produce the next generation. Random mutation ensures the evolution of the generation to successfully attain the specified goal. After the GA iterative loop is executed, the massive 
MIMO system can be calibrated automatically to attain the maximum EIRP and $\mathbf{W}_{\text {opt }}$ simultaneously.

Figure 3 illustrates numerical simulation results regarding the autocalibration of the 64-element MIMO system through a GA with a population $(M)$ of 20000 and 600 iterative loops $\left(I_{\mathrm{c}}\right)$. Figure $3(\mathrm{a})$ presents plots from a convergence analysis with respect to the iterative number, indicating the amplitude/phase standard deviation error of $\mathbf{W}$ to be less than $0.5 \%$ and antenna gain to be higher than $27.18 \mathrm{dBi}$ after 300 iterative loops. In Figure 3(a), the solid line, dashed line, and dashed line with circles represent the simulated antenna gain, amplitude error (\%), and phase error (\%), respectively. Figure 3(b) shows a plot of the computed EIRP against the observation angle $(\theta)$ under $\mathbf{W}$ before and after calibration, revealing that the computed EIRP can be improved from -30 to $-8.6 \mathrm{dBm}$ in the boresight direction after the GA-based calibration. The corresponding phase and normalized magnitude of $\mathbf{W}$ used in the EIRP computation (Figure 3(b)) are plotted in Figure 3(c). The marginal deviation in the phase/amplitude of the excitation coefficient can be attributed to the quantization error.

2.3. Modified GA for Far-Field Calibration. Generally, information regarding the randomness of the error of the excitation weighting vector is not available. In the previous section, to ensure the accuracy of the calibration results, we assume that the phase/amplitude of the error of weighting vectors is uniformly distributed in the interval $(0,2 \pi) /(0.05$, $0.2)$; moreover, we use a large population and high number of iterations in the GA optimization loops. This is a timeconsuming process and is unsuitable for mass production. If the randomness of the error of the vector can be adequately characterized and set in advance for the GA iterative loop, the calibration time can be reduced considerably.

Accordingly, the modified GA proposed in this study solves the aforementioned problem of high time complexity. In the modified GA, a large historical database with a reward mechanism is established for machine learning of the characteristics of random errors. Therefore, in mass production, the random behavior of each population can be estimated according to the parameters established in the historical database such that the calibration time can be reduced considerably. Figure 4 displays the flowchart of the modified GA. To assess the feasibility of the modified GA, we can use a preset Gaussian error of the vector $\left(\delta \mathbf{W}_{s}\right)$ with an amplitude $(\mathrm{v} / \mathrm{v})$ and phase ( $\mathrm{rad})$ mean/standard deviation of $m=0 / \sigma=0.05$ and $m=0 / \sigma=1.57$, respectively.

We intentionally ignore this background information and set the population of random genes $(M)$ with a uniform distribution of the phase/amplitude of the error of the vector in $(0,2 \pi) /(0,0.2)$ to 20000 ; moreover, we set number of iterative loops $\left(I_{\mathrm{c}}\right)$ to 500 . We execute the GA-based calibration process 60 times $(\mathrm{NL}=60)$. When the specified goal is achieved such that $\mathbf{W}_{\text {opt }}(i)(i=1, \ldots, \mathrm{NL})$, the error of the vector can be simply computed using formula $\delta \mathbf{W}(i)=\mathbf{W}_{\mathrm{opt}}$ $(i)-\mathbf{W}(i)$ to produce $3840(\mathrm{NL} \times N=64 \times 60)$ random amplitude/phase error numbers. For 3840 random variables, we can fit the probability distribution function of this event after running $60 \mathrm{GA}$ calibration procedures.

The simulation of the historical database after the 60 calibration runs indicates that the mean $(\mu)$ and standard deviation $(\sigma)$ of the amplitude/phase of the error vector $(\delta \mathbf{W})$ with a Gaussian distribution are $-0.0097 /-0.00117$ and $0.048 / 0.151$, respectively. Hence, we adaptively reset the estimation parameters ( $\mu$ and $\sigma$ ) for use in the iterative loops of our modified GA (Figure 4) to reduce the calibration time.

The convergence analysis results obtained from the simulation of our GA are plotted in Figure 5 and compared with those obtained using the conventional GA. The solid lines with symbols represent the simulated results for our modified GA and the dashed lines with solid symbols represent the results for the conventional GA.

Figure 5 reveals that our modified GA reduces the required number of iterative loops by 75 (from 275 to 200 loops), representing a nearly $27 \%$ reduction, compared with the conventional GA. A 3-byte SPI write operation for the transmission of write commands would require $1200 \mathrm{~ns}$ on the basis of a $20-\mathrm{MHz}$ clock rate; this would result in a latency of at least $76.8 \mathrm{~ms}$ for one beam-steering command as well as additional computation time. Therefore, the proposed GA would require at least 15.36 secs $(76.8 \mathrm{~ms} \times 200)$ to execute calibration processes.

\section{Experimental Study}

Because of budget limitations, we conducted a simple experimental study (Figure 6(a)) using a $2 \times 2$ subarray antenna module of the massive MIMO system (Figure $1(\mathrm{~b})$ ). In the experiment, four microstrip antennas were printed on the top-side and the front-end IC (IDT5280) associated with one I/O and one power connector mounted on the back side of the PCB.

Considering the lack of the dedicated testing software to command the front-end IC, we performed a bench test (Figure 6(b)) by using IDT's testing software (Timing Commander) to generate the database for calibration through the proposed method. The database included the amplitude/phase information of 64-phase shifter command states (6 bits) changing corresponding to each element at operational frequencies. On the basis of the bench test results, the signal vector error $(\mathbf{W})$ engendered by changes in the phase command states was simulated to calibrate the phase distribution of the $2 \times 2$ MIMO system using the proposed method (Section 2.2). We performed the calibration at a frequency of $27 \mathrm{GHz}$ only because the chipset vendor already provided the default setting of $28 \mathrm{GHz}$. To simplify the experiment, we ensured that all channels constantly radiated at the maximum power gain level. Table 1 lists the calibration data of phase shifter command states corresponding to the four-element system at 27 and $28 \mathrm{GHz}$. Running a bench test would require $2.5 \mathrm{~h}$; nevertheless, our proposed calibration method could shorten the duration of this time-consuming process to a few seconds subject to the availability of dedicated testing software. Subsequently, as depicted in Figure 7, we applied the default setting of the front-end IC $(f=28 \mathrm{GHz})$ for the experimental 


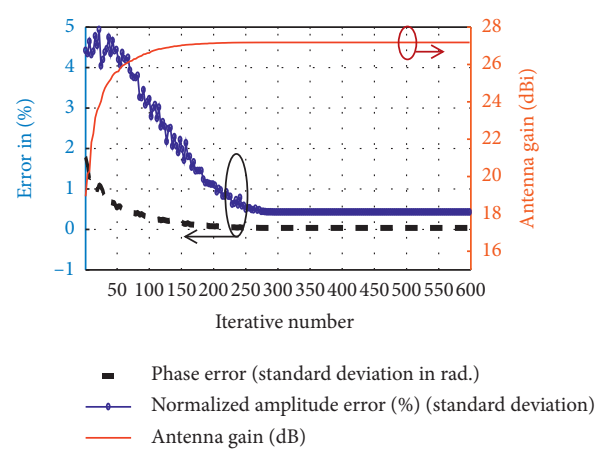

(a)

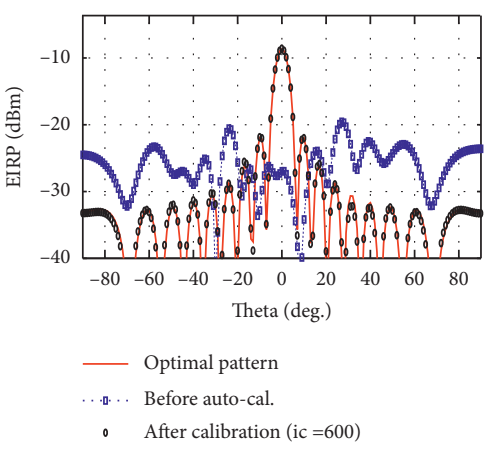

(b)

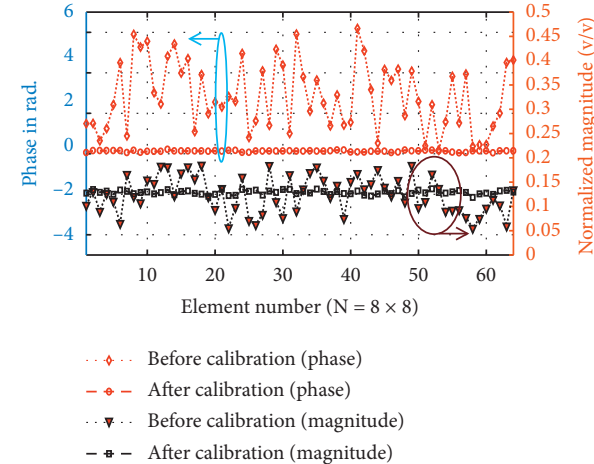

(c)

Figure 3: (a) Convergence analysis results regarding antenna gain and error of $\mathbf{W}$ against an iterative sequence number. (b) Simulated far-field patterns obtained after the use of GA $\left(M=20000\right.$ and $\left.I_{\mathrm{e}}=600\right)$. (c) Corresponding phase and normalized magnitude of the weighting vector $(\mathbf{W})$ used in EIRP computation.

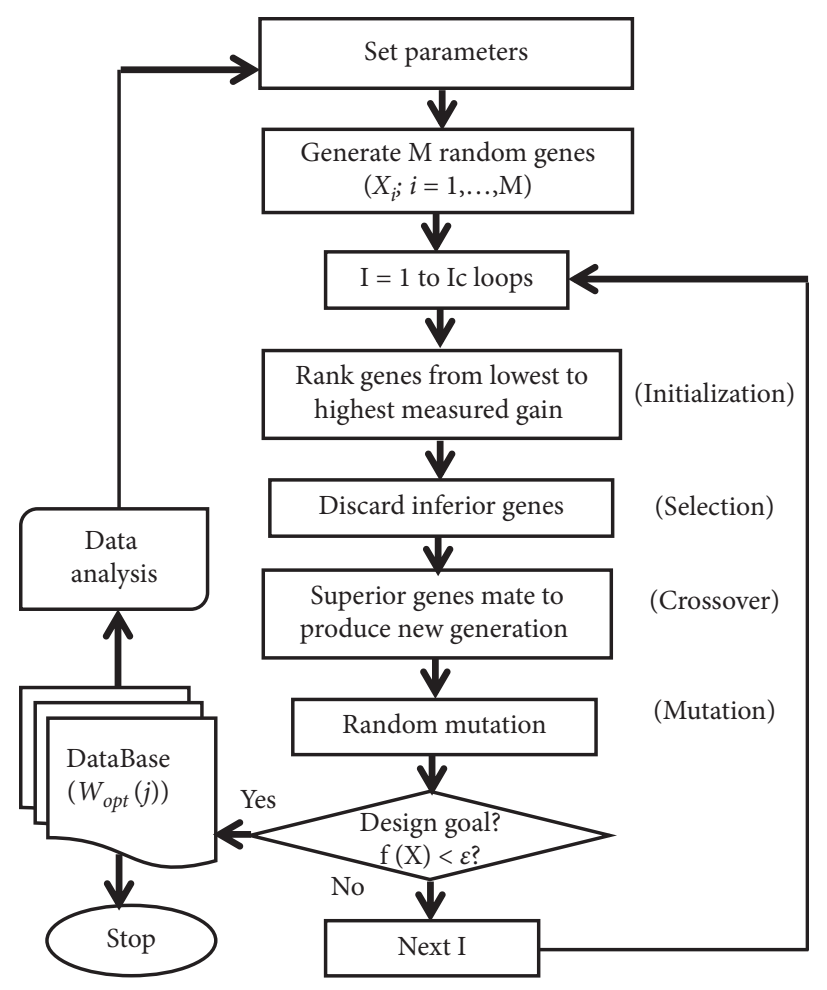

FIGURE 4: Flowchart of modified GA proposed in this study.

study by conducting OTA measurements at a compact antenna test range at Oriental Institute of Technology.

Figure 8 displays plots of the measured copolarization radiation patterns at $27 / 28 \mathrm{GHz}$, indicating a measured peak gain of $44.2 / 48.65 \mathrm{dBi}$. The measured patterns indicate the simultaneous achievement of both maximum peak gain and adequately calibrated phase distributions, as illustrated by the symmetrical radiation patterns in the boresight direction (Figure 9). We subsequently applied the data calibrated by the proposed method (Table 1 at $27 \mathrm{GHz}$ ) to the F5280 IC register to perform OTA measurements again. The measurement results demonstrated that the gain could be improved by $3.3 \mathrm{~dB}$ at $27 \mathrm{GHz}$ but degraded by $2.85 \mathrm{~dB}$ at $28 \mathrm{GHz}$, indicating that excitation coefficient calibration against operating frequencies is essential for MIMO system performance.

Figure 9 illustrates plots of the measured EIRP and TRP against the input power level, with the power being swept from 0 to $10 \mathrm{dBm}$ at VNA and being attenuated by approximately $34.5 \mathrm{~dB}$ cable loss. Finally, we changed the phase shifter states to be out of phase along the $x$-axis to form difference patterns. The measurement results (Figure 10) 


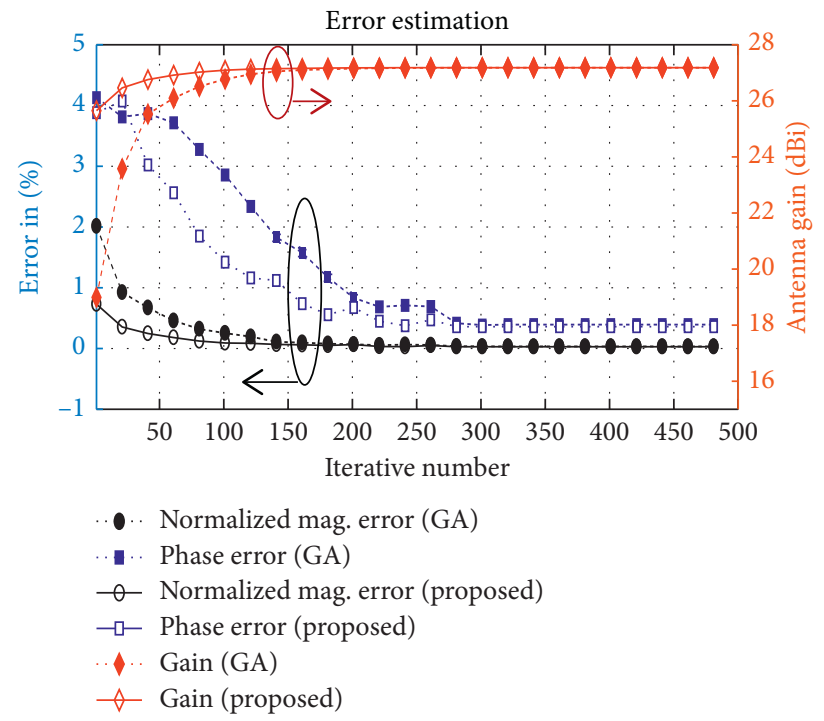

FIGURE 5: Comparison of convergence analysis results between conventional and modified GAs (Figure 2).

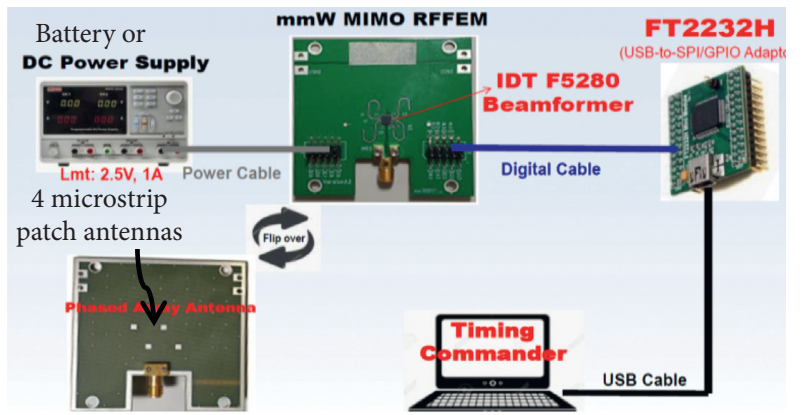

(a)

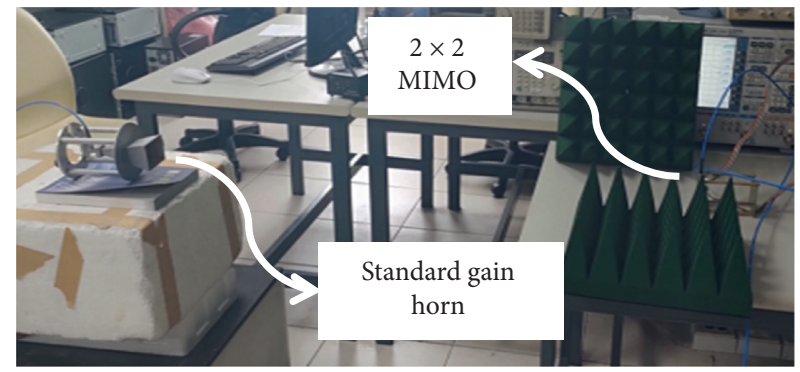

(b)

Figure 6: (a) Illustration of $2 \times 2$ MIMO antenna module; (b) photograph of bench test conducted to generate calibration database.

TABLE 1: Calibration results.

\begin{tabular}{|c|c|c|c|c|c|c|c|c|}
\hline \multirow{2}{*}{ Freq. (GHz) } & \multicolumn{2}{|c|}{ Channel 1} & \multicolumn{2}{|c|}{ Channel 2} & \multicolumn{2}{|c|}{ Channel 3} & \multicolumn{2}{|c|}{ Channel 4} \\
\hline & Am. $(\mathrm{dB})$ & State/phase & Am. $(\mathrm{dB})$ & State/phase & Am. $(\mathrm{dB})$ & State/phase & Am. (dB) & State/phase \\
\hline 28 & -17.8 & $0 / 62.6^{\circ}$ & -15.7 & $0 / 50.7^{\circ}$ & -15.5 & $0 / 59.4^{\circ}$ & -16.1 & $0 / 61.4^{\circ}$ \\
\hline 27 & -17.4 & $27 / 44^{\circ}$ & -15.4 & $23 / 60^{\circ}$ & -18.2 & $23 / 71^{\circ}$ & -18.3 & $25 / 55^{\circ}$ \\
\hline
\end{tabular}

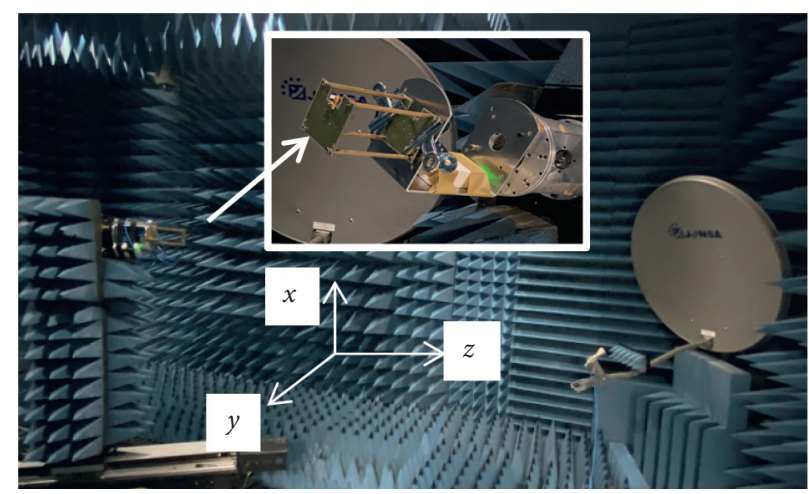

FIGURE 7: OTA measurements in CATR chamber at Oriental Institute of Technology. 


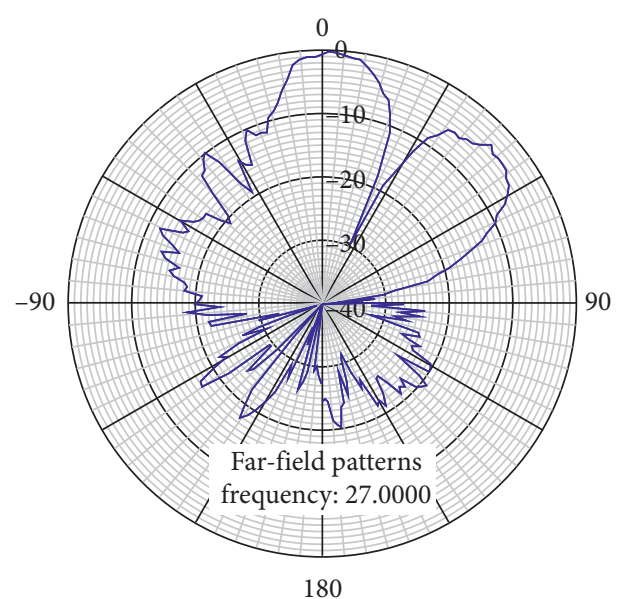

(a)

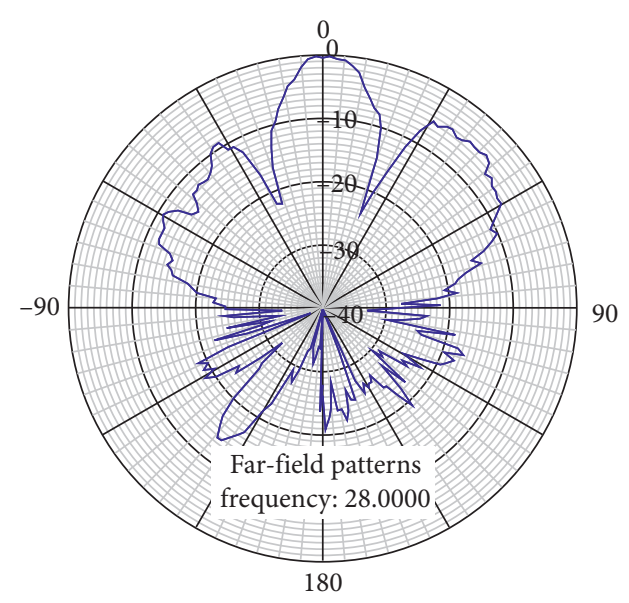

(b)

Figure 8: Copolarized radiation patterns in $y-z$ plane at (a) $f=27 \mathrm{GHz}$ and (b) $f=28 \mathrm{GHz}$.

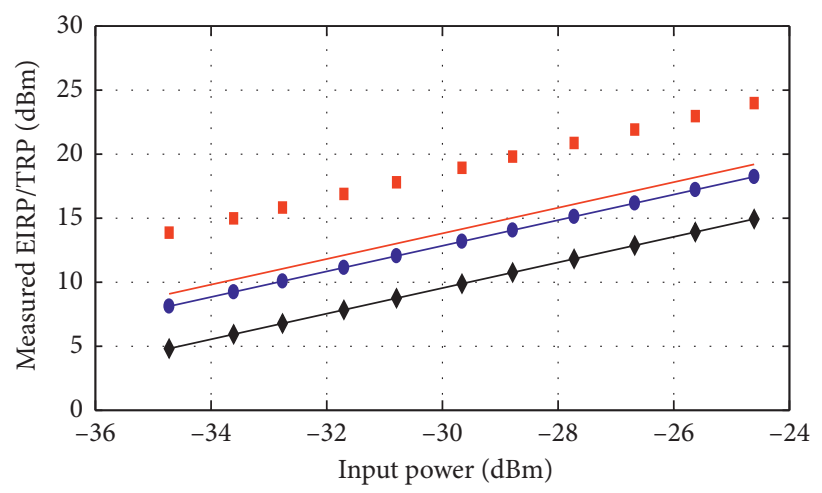

- Measure $\operatorname{EIRP}(f=28 \mathrm{GHz}$ with calibration)

$\rightarrow$ Measured TRP $(f=27 \mathrm{GHz}$ with calibration $)$

- Measured TRP $(f=28 \mathrm{GHz}$ with calibration)

$\multimap$ Measured TRP $(f=27 \mathrm{GHz}$; without calibration)

Figure 9: Measured EIRP and TRP (in dBm).

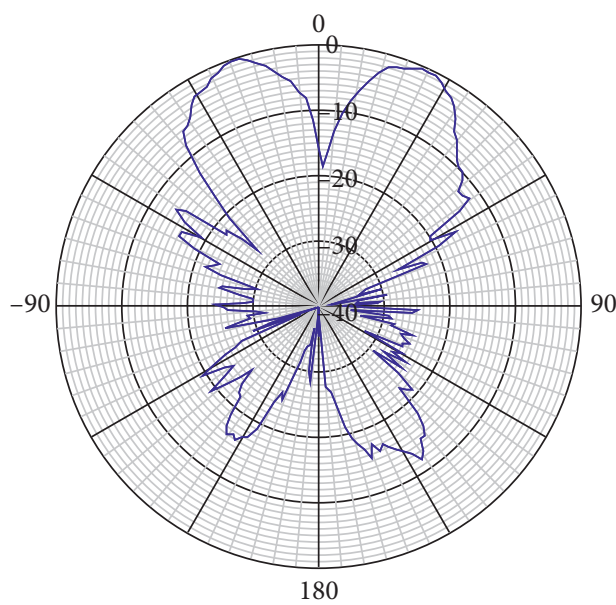

(a)

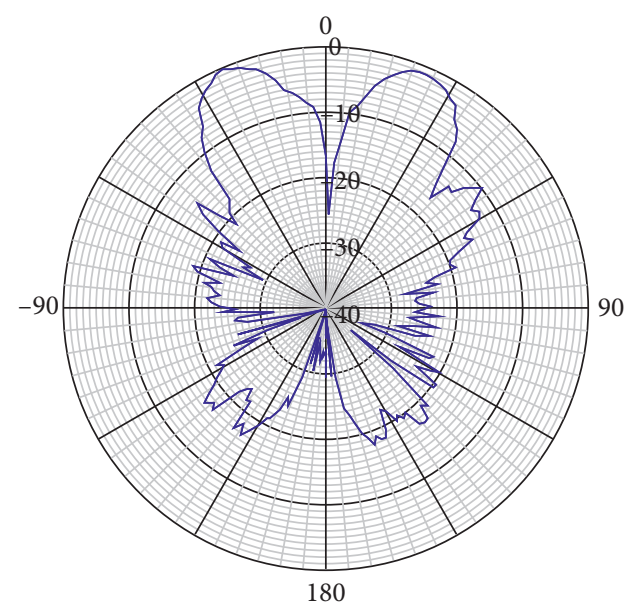

(b)

Figure 10: Copolarized radiation pattern (DIF.) in $y$ - $z$ plane at (a) $f=27 \mathrm{GHz}$ and (b) $f=28 \mathrm{GHz}$. 
confirmed the beam-steering capability of the MIMO antenna module that was adequately calibrated using the proposed method.

\section{Conclusion}

The deployment of the mm-wave massive MIMO in the form of a small cell or customer-provided equipment is essential for 5G applications. Reliable and time-efficient calibration and test methods are crucial for developing $\mathrm{mm}$ wave massive MIMO systems. In this study, we developed an $8 \times 8$ massive MIMO system with a triangular lattice to achieve a theoretical maximum EIRP of $56.26 \mathrm{dBm}$. We first propose a modified GA for the far-field calibration of the $\mathrm{mm}$-wave massive MIMO system. We then present a numerical analysis to validate the effectiveness of the proposed GA. Finally, we executed a simple experiment to validate that a well-calibrated phase distribution can enable the achievement of maximum array gain in the boresight direction. Therefore, our proposed method can enable automatic calibration of massive MIMO systems [19].

\section{Data Availability}

The figures' data used to support the findings of this study are currently under embargo while the research findings are commercialized. Requests for data, 12 months after publication of this article, will be considered by the corresponding author.

\section{Conflicts of Interest}

The authors declare that they have no conflicts of interest.

\section{Acknowledgments}

This study was financially supported by FIH Mobile Limited. The authors thank the FIH team for supporting the development of the design project.

\section{References}

[1] 5G, Vision White, Paper-Samsung (2015), https://www. samsung.com/global/business/networks/insights/5g-vision/.

[2] C.-N. Hu, "A novel method for calibrating deployed active antenna arrays," IEEE Transactions on Antennas and Propagation, vol. 63, no. 4, pp. 1650-1657.

[3] R. Sorace, "Phased array calibration," IEEE Transactions on Antennas and Propagation, vol. 49, no. 4, pp. 517-525, 2001.

[4] R. Long, J. Ouyang, F. Yang, W. Han, and L. Zhou, "Fast amplitude-only measurement method for phased array calibration," IEEE Transactions on Antennas and Propagation, vol. 65, no. 4, pp. 1815-1822, 2017.

[5] R. Long, J. Ouyang, F. Yang, W. Han, and L. Zhou, "Multielement phased array calibration method by solving linear equations," IEEE Transactions on Antennas and Propagation, vol. 65, no. 6, pp. 2931-2939, 2017.

[6] W. T. Patton and L. H. Yorinks, "Near-field alignment of phased-array antennas," IEEE Transactions on Antennas and Propagation, vol. 47, no. 3, pp. 584-591, 1999.

[7] H. M. Aumann, A. J. Fenn, and F. G. Willwerth, "Phased array antenna calibration and pattern prediction using mutual coupling measurements," IEEE Transactions on Antennas and Propagation, vol. 37, no. 7, pp. 844-850, 1989.

[8] T. Sato and R. Konho, "New calibration matrix calculation method for removing the effect of mutual coupling for uniform linear arrays," in Proceeedings of the IEEE 63rd Vehicular. Technology Conferences, pp. 2686-2690, Melbourne, Australia, May 2006.

[9] K.-M. Lee, R.-S. Chu, and S.-C. Liu, "A built-in performancemonitoring/fault isolation and correction (PM/FIC) system for active phased-array antennas," IEEE Transactions on Antennas and Propagation, vol. 41, no. 11, pp. 1530-1540, 1993.

[10] Transmit/receive half duplex IC $25 \mathrm{GHz}$ to $31 \mathrm{GHz}$, https:// www.idt.com/us/en/products/rf-products/phased-arraybeamformers/f5280-transmitreceive-half-duplex-ic-25ghz31ghz.

[11] W. Von aulock, "Properties of phased arrays," in Proceeedings of the IRE 190 (Reprinted In Hansen, R. C. Significant Phased Array Papers, Artech House, Norwood, MA, USA, October 1973.

[12] Q. J. Zhang, K. C. Gupta, and V. K. Devabhaktuni, “Artificial neural networks for RF and microwave design- from theory to practice," IEEE International Microwave Symposium, vol. 51, no. 4, pp. 1339-1350, 2003.

[13] Y. Wang, M. Yu, H. Kabir, and Q. J. Zhang, "Effective design of cross-coupled filter using neural networks and coupling matrix," in Proceedings of the IEEE International Microwave Symposium, pp. 1431-1434, San Francisco, CA, USA, June 2006.

[14] F. Farbiz and M. B. Menhaj, "A fizzy logic control based approach fop image filtering," in Fuzzy Techniques In Lmage Processing, E. E. Kerre and M. Nachtegael, Eds., pp. 194-221, Springer-Verlag, Berlin, Germany, 2000.

[15] J. Redvik, "Simulated annealing optimization applied to antenna arrays with failed elements,"IEEE Antennas And Propagation Society International Symposium, Orlando, FL, USA, July 1999.

[16] Y. Yang, Y. Tan, R. Liang, Q. Wang, and N. Yuan, "A calibration algorithm of millimeter-wave sparse arrays based on simulated annealing," in Proceedings of the 2010 International Conference Microwave and Millimeter Wave Technology, pp. 1703-1705, Chengdu, China, May 2010.

[17] S. H. Son, S. Y. Eom, S. I. Jeon, and W. Hwang, "Automatic phase correction of phased array antennas by a genetic algorithm," IEEE Transactions on Antennas and Propagation, vol. 56, no. 8, pp. 2751-2754, 2008.

[18] K. N. Sherman, "Phased array shaped multieam optimization for LEO satellite communications using a genetic algorithm," in Proceedings 2000 IEEE International Conference on Phased Array Systems and Technology, Dana Point, CA, USA, June 2000.

[19] K. Chellapilla and A. Hoorfar, "Evolutionary programming: an efficient alternative to genetic algorithms for electromagnetic optimization problems," in Proceedings of the AP. Society International Symposium, Atlanta, GA, USA, June 1998. 